\title{
Iloprost for asthma
}

\author{
Friedrich C. Luft
}

Asthma is a chronic inflammatory airway disease that is characterized by reversible episodes of bronchospasm related to hyper-responsiveness of bronchial smooth muscle. Copious mucous secretions and edema formation resulting from generalized inflammation accompany the bronchospasm. The incidence and prevalence of asthma in industrialized countries is very high and asthma is on the steady increase in the developing world [1]. The currently available treatments are suboptimal and additional, more effective treatments would be most welcome.

Iloprost is a synthetic prostacyclin PGI2 analog that dilates systemic and pulmonary arterial vascular beds. In this issue, Kuo et al. report on the effects of iloprost on $\mathrm{T}$ helper (Th)1- and Th2-related chemokines that may play a role in asthma pathogenesis [2]. The authors prepared a monocyte cell line and treated the cells with iloprost and treprostinil, another PGI2 analog. Then, the cells were stimulated with lipopolysaccharide (LPS). Macrophagederived chemokine (MDC) and interferon- $\gamma$-inducible protein-10 (IP-10) were monitored and signal transduction pathways were observed. The authors found that the PGI2 analogs enhanced MDC production but suppressed IP-10 expression in LPS-stimulated cells. They employed an Iprostanoid receptor antagonist, peroxisome proliferative activated receptor (PPAR) $\alpha$ and $\gamma$ antagonists, and forskolin, in further studies. The I-prostanoid receptor blocker abrogated the effects of the PGI analogs on MDC production and IP-10 suppression. The PPAR antagonists were similarly inhibitory of the PGI2 analogs-related effects. The authors found that the PGI2 analogs stimulated cyclic AMP levels in their cells and observed that a similar

F. C. Luft $(\bowtie)$

Experimental and Clinical Research Center, Max-Delbrück Center for Molecular Medicine and Charité Medical Faculty,

Berlin, Germany

e-mail: luft@charite.de
MDC stimulation and IP-10 suppression could be achieved by stimulating andenylate cyclase with forskolin. The authors also found that by inhibiting the mitogen-activated protein kinase $\mathrm{p} 38$ or inhibiting the nuclear factor kappa-B, the effects of the PGI2 analogs could be reduced. The authors further showed that iloprost enhanced $\mathrm{H} 3$ acetylation in the MDC promoter area and suppressed H3 acetylation in the IP-10 promoter area. Kuo et al. conclude that PGI2 analogs increase MDC expression via the Iprostanoid receptor and that stimulation of the receptor generates cyclic AMP. The pathway involves PPAR- $\alpha$, PPAR- $\gamma, \mathrm{NF \kappa B}, \mathrm{p} 38$, and the activating transcription factor-2 (ATF2). The ATF2 protein is also a histone acetyltransferase that could activate transcription by direct effects on chromatin components. The authors implicate ATF2 in the enhanced acetylation of the MDC promoter. IP-10 was suppressed by the PGI2 analogs via pathways mediating reduced methyltransferase activity. As a result, PGI2 analogs may therefore increase Th2 recruitment and inflammation. These results would suggest that PGI2 analogs could make asthma worse instead of better.

Firstly, we should examine Th1 and Th2 immunobalance. Th cells are lymphocytes that cannot kill infected host cells or pathogens. They would be fairly useless, were it not for their activation and direction of other immune cells. Th cells express the surface protein CD4 and the T cell receptor/CD3 complex. Th lymphocytes are activated by a complex two-signal activation. Once activation is complete, the Th cells proliferate by releasing a potent $T$ cell growth factor, interleukin (IL-2), which also acts in an autocrine fashion. The cells can differentiate forming Th0 cells secreting IL-2, IL-4, and interferon- $\gamma$ (IFN- $\gamma$ ), Th1 cells that produce primarily IL- 2 and IFN- $\gamma$, or Th 2 cells, driven by IL-4. The Th2 cells produce IL-3, IL-4, IL-10, and IL-13. The Th cell-balance model has been expanded to include other components, such as Th17 and T regulatory (Treg) cells. Tilting the balance towards Th2 cells could 
mean that IL-4 gains an upper hand. IL-4 could stimulate B lymphocytes to produce more $\mathrm{IgE}$ and $\mathrm{IgG} 4$. Th2 reactions are important in allergic and parasitological diseases, both relevant to asthma.

Chemokines are a family of small cytokines-like proteins secreted by cells. They commonly induce chemotaxis in nearby responsive cells, hence the term chemotactic cytokines (CC). MDC, better known as CCL22, is a CC chemokine [3]. MDC is constitutively expressed; however, microbial products (LPS) and cytokines influence the expression. Th2 (IL-4 and IL-13) and Th1 (interferon) cytokines have divergent effects. MDC acts on various target cells including dendritic cells, natural-killer cells, and other $\mathrm{T}$ cell subsets. MDC is a potent attractant for CCR4 expressing polarized Th2 cells. MDC could play a role as an amplification loop of Th2 responses. Kuo et al. [2] found that PGI2 analogs increased MDC expression in the LPSstimulated monocytes.

IP-10 is a CXC or an $\alpha$-chemokine. The protein is also known as CXCL10; the receptor is CXCR3. IP-10 functions primarily as a chemo-attractant and as a monocyte and $\mathrm{T}$ lymphocyte activator. IP-10 can also influence cell growth and function as a chemo-attractant to promote lymphocyte adhesion to endothelial cells. Thus, IP-10 is an inducible protein that participates in directional cell migration in inflammatory disease states and vascular remodeling. Kuo et al. [2] found that PGI2 analogs diminished IP-10 expression in LPS-treated monocytes.

The authors argue that enhanced MDC expression in the face of reduced IP-10 expression could cause problems, presumably related to amplification of increased Th2 responses via the MDC amplification loop and decreased suppression of cell proliferation via reduced activity of the CXCL10/CXCR3 system [4]. They argue for caution, which is surely warranted. However, cell-based in vitro studies also have their weaknesses. Kuo et al. [2] relied on a human monocytic cell line (THP-1 cells), although in one experiment, human peripheral blood mononuclear cells were also used. The term "monocyte" implies a single, homogenous cell population with a uniform physiology. However, monocytes differentiate further into macrophages as they leave the blood and enter the tissue. Macrophages are currently the focus of intensive research activities. We are dealing with a heterogeneous cell type, which in its various forms exerts completely different functions. Currently, macrophages are subdivided in classically activated, wound-healing, and regulatory macrophages (Fig. 1).

Classically activated macrophages arise upon IFN- $\gamma$ stimulation. Th1 cells, $\mathrm{CD} 8^{+} \mathrm{T}$ cells, and natural-killer cells, typically produce IFN- $\gamma$ during innate immune responses or upon antigen presenting-cell produced TNF$\alpha$ stimulation. Wound-healing (or alternatively activated) macrophages arise in response to IL-4. Th2 cells and

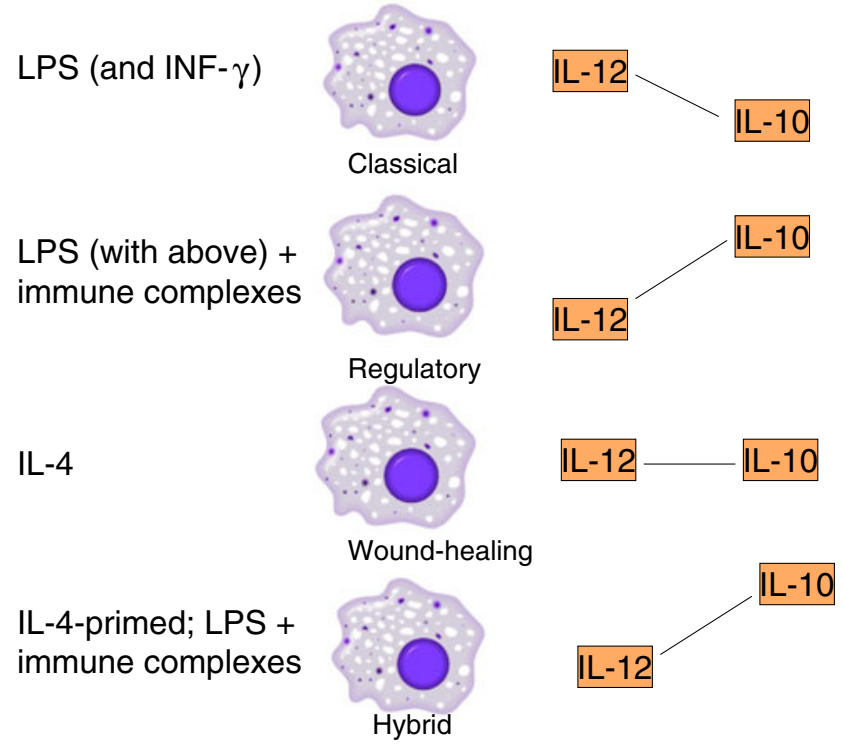

Fig. 1 They may all look the same, but they are not. Classically activated macrophages produce high levels of interleukin (IL)-12 and modest levels of IL-10. Regulatory macrophages produce more IL-10 and less levels of IL-12. IL-4 induces macrophages (wound-healing macrophages) to produce low levels of these both but also other proteins. IL-4-primed macrophages exposed to LPS and immune complexes results in a hybrid phenotype in with features of regulatory and wound-healing macrophages

neutrophils can produce IL-4. Regulatory macrophages are generated in response to various stimuli, including immune complexes, prostaglandins, G-protein coupled-receptor ligands, glucocorticoids, apoptotic cells, or IL-10. Each of these three macrophage populations has a distinct physiology. Classically activated macrophages have proinflammatory activity, whereas regulatory macrophages produce high levels of IL-10 and low levels of IL-12 to suppress immune responses. IL-10 can render macrophages refractory to the activating effects of IFN- $\gamma$. IL-10 can also bias T cells to produce IL-4 and IL-10. Wound-healing macrophages are similar to the previously described alternatively activated macrophages and have a role in tissue repair [5]. Kuo et al. [2] emphasize the importance of macrophages. Whether or not in vitro studies on a single monocyte cell line reflects pathophysiology in an intact organism must be investigated in vivo.

As Kuo et al. [2] duly cite in their paper, iloprost has been studied in an animal asthma model. In that study, iloprost performed better. Idzko et al. [6] studied a mouse model of asthma. The mice were treated with the PGI2 analog (iloprost), with the notion that the compound would act mainly as a smooth muscle dilator via the I-prostanoid receptor. However, iloprost interfered with the function of myeloid dendritic (antigen presenting) cells. The group found that iloprost treatment inhibited the maturation and migration of pulmonary dendritic cells to mediastinal 
lymph nodes. As a result, induction of an allergen-specific Th2 response in these nodes was attenuated. As a matter of fact, the effect of iloprost was dendritic-cell autonomous, as iloprost-treated dendritic cells no longer induced Th2 differentiation from naive $\mathrm{T}$ cells or boosted effector cytokine production in primed Th2 cells. These in vivo results would suggest that the concerns, regarding prostaglandin as a therapeutic target as raised by the Kuo et al. [2] paper, should be interpreted in context. Kuo et al. showed novel epigenetic signaling via acetylation enhancement and suppression of their target molecules [2]. This signaling discovery must be taken into strong consideration in future studies of immune cell regulation.

Müller et al. report that iloprost has potent antiinflammatory properties on human monocyte-derived dendritic cells [7]. They found that human monocyte-derived dendritic cells expressed the I-prostanoid PGI2 receptor. Stimulation with iloprost increased cyclic AMP levels but inhibited the secretion of TNF- $\alpha$, IL-6, IL-8, and IL-12 while IL-10 production was enhanced. In co-culture experiments of iloprost-treated dendritic and naive CD45RA $+\mathrm{T}$ cells, the authors found induction of regulatory $\mathrm{T}$ cells, as demonstrated by increased intracellular FoxP3 expression and IL-10 production. Iloprost also inhibited the macrophage inflammatory protein-3-beta-induced dendritic cell migration.

I am not aware of any studies looking at patients in response to iloprost. There are utilitarian human models. A bronchial challenge test assists in the diagnosis of asthma. The patient breathes in nebulized methacholine or histamine and placebo. Both drugs provoke bronchoconstriction. Histamine also causes nasal and bronchial mucus secretion and bronchoconstriction via the $\mathrm{H} 1$ receptor. Methacoline utilizes the M3 receptor for bronchial constriction. The degree of narrowing can then be quantified by spirometry. The idea would be whether or not iloprost interferes with bronchoconstriction acutely or chronically. I believe we will have to wait until human studies settle this issue.

Respectfully,

Friedrich C. Luft

\section{References}

1. Eder W, Ege MJ, von Mutius E (2006) The asthma epidemic. N Engl J Med 355:2226-2235

2. Kuo C-H, Ko Y-C, Yang S-N, Chu Y-T, Wang W-L, Huang S-K, Chen H-N, Wei W-J, Jong Y-J Hung C-H (2010) Effects of PGI2 analogues on Th1- and Th2-related chemokines in monocytes via epigenetic regulation. J Mol Med. doi:10.1007/s00109-010-0694-2

3. Mantovani A, Gray PA, Van Damme J, Sozzani S (2000) Macrophage-derived chemokine (MDC). J Leukoc Biol 68:400404

4. Shimada A, Oikawa Y, Yamada Y, Okubo Y, Narumi S (2009) The role of the CXCL10/CXCR3 system in Type 1 diabetes. Rev Diabet Stud 6:81-84

5. Mosser DM, Edwards JP (2008) Exploring the full spectrum of macrophage activation. Nat Rev Immunol 8:958-969

6. Idzko M, Hammad $\mathrm{H}$, van Nimwegen $\mathrm{M}$, Kool M, Vos $\mathrm{N}$, Hoogsteden HC, Lambrecht BN (2007) Inhaled iloprost suppresses the cardinal features of asthma via inhibition of airway dendritic cell function. J Clin Invest 117:464-472

7. Müller T, Dürk T, Blumenthal B, Herouy Y, Sorichter S, Grimm M, Panther E, Cicko S, Norgauer J, Idzko M (2010) Iloprost has potent anti-inflammatory properties on human monocyte-derived dendritic cells. Clin Exp Allergy 40:1214-1221 